\title{
A new model for surface potential decay of corona-charged polymers
}

\author{
George Chen \\ School of Electronics and Computer Science, University Of Southampton, Southampton, SO16 1BJ, UK \\ E-mail: gc@ecs.soton.ac.uk
}

Received 10 September 2009, in final form 24 November 2009

Published 21 January 2010

Online at stacks.iop.org/JPhysD/43/055405

\begin{abstract}
Surface potential measurement provides a useful tool to gauge the electrical properties of materials. It has been observed that the potential of a sample with an initial high surface potential decays faster than that with an initial lower surface potential, known as the cross-over phenomenon. The phenomenon was found a few decades ago and various theories and models have been proposed. A common feature of the existing models is based on single charge carrier injection from a corona-charged surface. With our recent space charge measurement results on corona-charged samples, double injection from both electrodes has been verified. Based on this new fact, a new model based on bipolar charge injection is proposed and initial numerical simulation reveals that the surface potential cross-over phenomenon can occur under bipolar charge injection.
\end{abstract}

(Some figures in this article are in colour only in the electronic version)

\section{Introduction}

Over the years, considerable interest has been shown in the surface potential decay of corona-charged polymeric materials. In its most basic form, a surface potential experiment involves charging the surface of an insulating material to a voltage $V_{0}$. The charging is most conveniently achieved by a corona device which can deposit charges of positive or negative polarity onto the surface of the material. Following the charging process, the time dependence of the surface potential $V(t)$ can be monitored via an electrostatic voltmeter. The measurement of the potential decay has been proven to be a simple and useful technique for characterizing insulating materials and the charging method. For example, this method enables a convenient determination of charge carrier mobility and trap parameters. One of the well-known effects in the observation of surface potential decay is the cross-over phenomenon [1], i.e. initially the surface potential of a sample charged to a highpotential decays more rapidly than one charged to a lower potential. Most of the theories addressed the time evolution of the surface potential in terms of surface conduction [2], charge injection [3-5] and polarization [6]. The recent literature on potential decay measurement is dominated by the hypothesis of injection into the bulk of the charge deposited on the surface accompanied by slow polarization processes within the bulk under the influence of the deposited charge. Clearly, a thorough understanding of the detailed physical process of the kinetics of surface potential decay is required.

With the recent progress in space charge measurement techniques, it is possible to observe charge evolution within the bulk of the corona-charged insulating material. Our papers $[7,8]$ demonstrated that bipolar charge injection has taken place in the low density polyethylene (LDPE) samples during and after corona charging using the pulsed electroacoustic technique (PEA). In the light of this new experimental evidence, we propose a new model which incorporates charge injection from the top and bottom surfaces and field-dependent mobility.

\section{Surface potential decay}

A typical corona charging experimental setup is shown in figure 1 . The surface potential of the sample will be the same as the grid voltage providing the potential difference between the needle and the grid is sufficiently high that the electric field around the needle is greater than the breakdown strength of air.

Once the corona charging stops, the surface potential starts to decay. The decay takes various forms depending on the corona charging conditions such as the grid voltage, charging time and sample thickness. Figure 2 shows a typical potential 


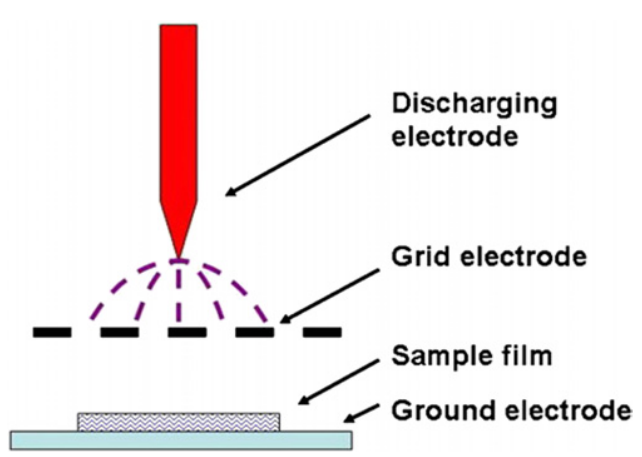

Figure 1. Schematic diagram of the corona-charging setup.

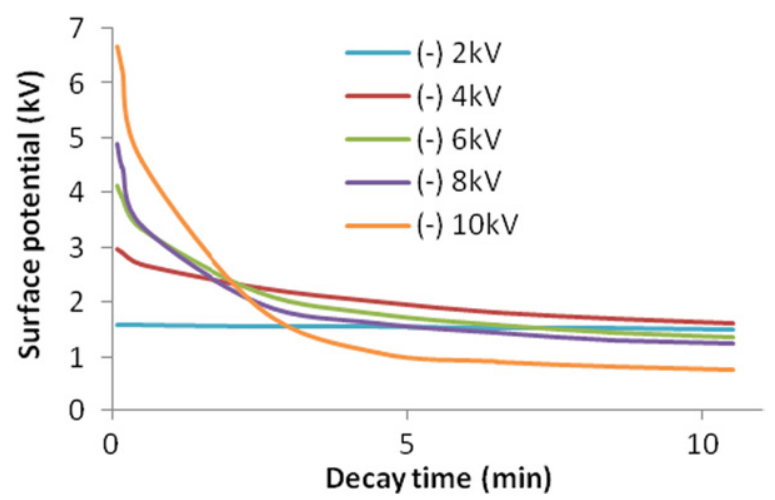

Figure 2. Potential decay for a $50 \mu \mathrm{m}$ LDPE sample for different corona voltages.

decay observed in LDPE polymeric materials when the grid voltage is changed. The charging time was 2 min for all the samples. Negative surface potential was obtained, however, for easy graphing, the absolute surface potential is used in this paper.

It has been observed that the potential of sample with initial high surface potential decays faster than that with initial lower surface potential, known as the cross-over phenomenon [1]. It can be seen that the cross-over point moves towards shorter times with an increase in charging voltage. Although the phenomenon was found a few decades ago and many efforts have been made to investigate the mechanisms, a satisfactory explanation has not been found so far.

It has also been noted that charging time also has a significant influence on the surface potential decay when the grid voltage is kept constant. Figure 3 illustrates a typical surface potential decay showing the influence of the charging time.

It can be seen that the surface potential of the sample with a longer charging time has a fast rate of decay compared with that of the sample with a shorter charging time.

\section{The existing models}

The surface potential decay and the cross-over phenomenon have been observed in various polymers $[9,10]$ and several models and theories have been proposed. Batra et al [11-13] assumed field-dependent mobilities and negligible penetration depth of the surface charge but neglected the

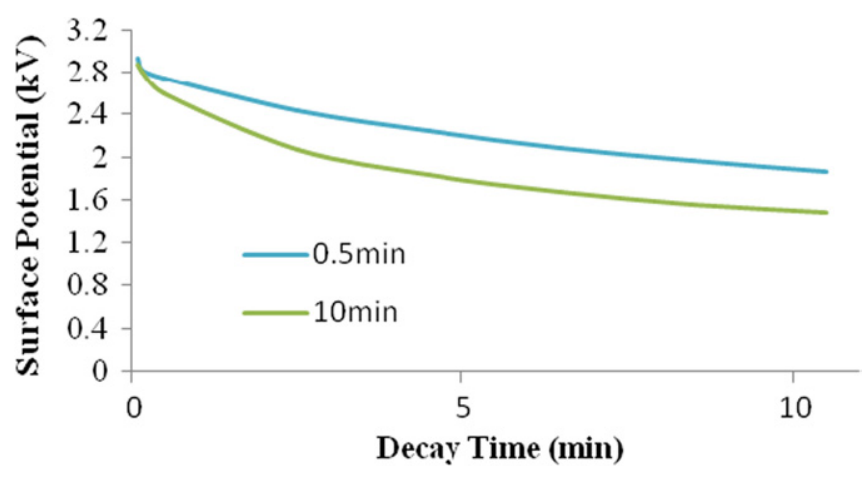

Figure 3. Surface charge decay in a $50 \mu \mathrm{m}$ LDPE sample corona charged at $4 \mathrm{kV}$.

effect of partial instantaneous injection and trapping. Wintle, in his papers [14-16], developed theories that include fielddependent mobilities of various forms as well as trapping but none of them explained the cross-over effect. Wintle also made an assumption that the depth of penetration of the initial charge is field independent. Batra proved that Wintle's theories also cannot account for the cross-over [17]. Later, Sonnonstine and Perlman came out with two distinct theories in their work [10]. The first is the modification of Batra's theory to include both instantaneous partial injection and field-dependent mobility. The second theory assumes time-dependent detrapping of charge carriers at the coronacharged surface. Although the approximate forms of the decay curves were theoretically predicted in the paper, they were not satisfactory as the charging conditions were neglected.

Baum et al $[18,19]$ were able to demonstrate that the crossover phenomenon depended upon the sign and the duration of the corona charging process. In their papers, it was shown that the cross-over phenomenon did not occur for positive corona voltage, which apparently contradicts the findings of Ieda et al $[1,20]$. They suggested that the excited molecules and photons generated in the corona-discharge process caused the charge which originally deposited in the deep surface to be injected into the bulk where it becomes mobile, thus increasing the decay rate of the surface potential especially for high initial potential. They were also able to prove that cross-over is charging-time dependent by demonstrating that no cross-over appeared for charging times of less than $\sim 25 \mathrm{~ms}$. The proposal of Baum and co-workers was then supported by the work of Kao et al [21] who revealed a deep surface trap distribution centred at $95^{\circ} \mathrm{C}$ and a shallow surface bulk distribution centred at $55^{\circ} \mathrm{C}$ on negatively corona-charged LDPE by using the thermally stimulated discharge technique. Using the same technique, they were able to show that the trapped charge in the shallow surface can be released by exciting molecules from the corona discharge. In 1980, Toomer and Lewis [22] introduced the existence of both deep and shallow surface traps in the sample. They also showed that negative charges penetrate more readily into the bulk and the bulk traps exist for both sign of carriers.

In addition to various assumptions which were not evident, one of the common features in the models proposed so far is that all the models are based on single charge carrier injection. 


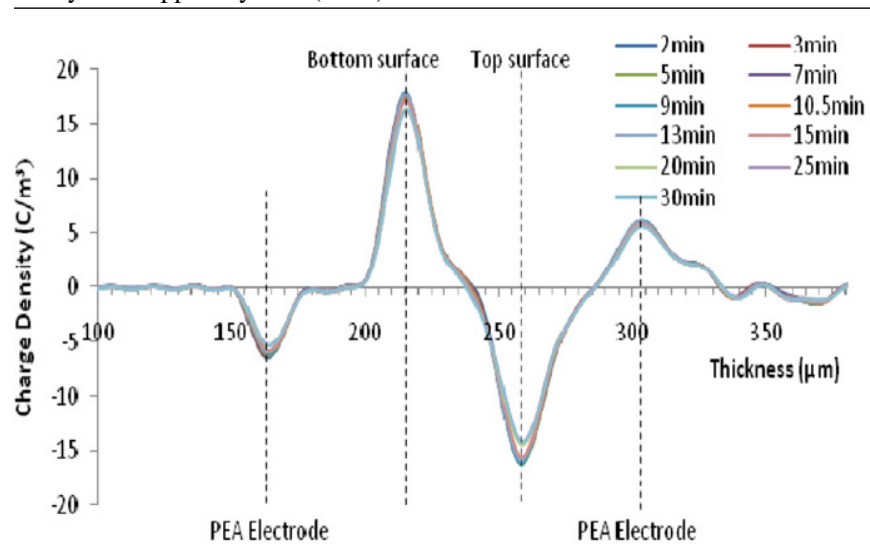

(a) $t_{c}=2$ minutes

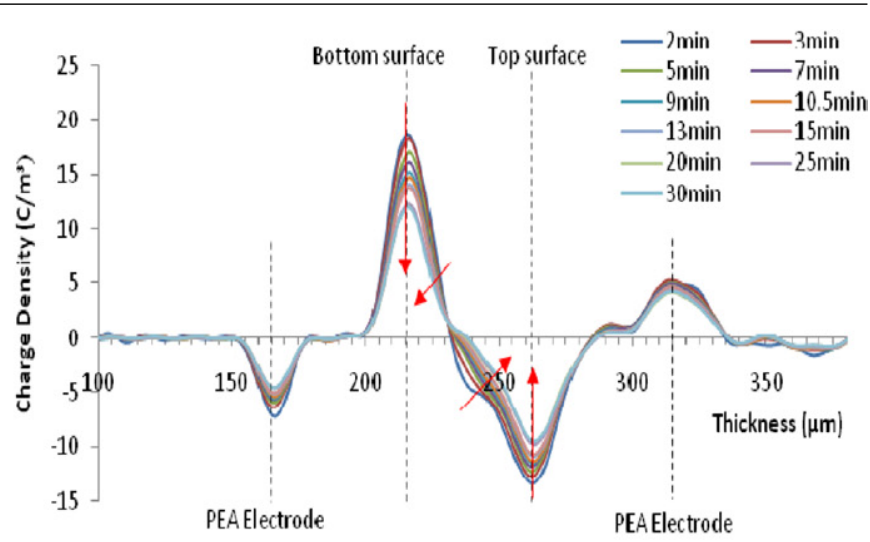

(b) $t_{c}=10$ minutes

Figure 4. Space charge distribution in a $50 \mu \mathrm{m}$ sample corona charged at $-2 \mathrm{kV}$ for $(a) 2 \mathrm{~min}$ and $(b) 10 \mathrm{~min}$.

Our new experimental evidence has shown this is not always the case especially where the cross-over is concerned. Bipolar charge injection has been verified by the measurement of space charge in the corona-charged sample as shown in the section below. This new finding challenges the existing surface potential decay models which were developed based on a single charge carrier injection.

\section{New evidence}

In the last two decades, significant progress has been made in developing techniques to map space charge in solid dielectrics. The PEA is one of the methods widely used. The author is one of the few people who used the technique to measure space charge in corona-charged polymeric materials [7]. It has been confirmed without any doubt that bipolar charge injection takes place during charging. For a typical sample, the presence of bulk space charge in the PEA results is observed through the charge profile or peak between the two electrode charge peaks. However, for a thin sample bulk charge profile or peak may overlap with the two electrode charge peaks; consequently, the presence of the bulk charge can be identified by the changes in the two electrode charge peaks, i.e. broadening or narrowing depending on the polarity of the bulk charge.

Figure 4 shows the space charge measurement in a $-2 \mathrm{kV}$ corona-charged LDPE film. To minimize the disturbance to the deposited charge, the top and bottom surfaces of the samples were protected by an extra layer of $50 \mu \mathrm{m}$ LDPE film. There are four distinctive charge peaks present across the sample from left to right. The first and the fourth peaks are known as induced charge peaks on the PEA electrodes. The presence of the other two peaks is due to the existence of charge in the sample. The second peak corresponds to the bottom surface of the $50 \mu \mathrm{m}$ corona-charged film and the third peak to the top surface. The third negative peak is expected as the sample was exposed to negative corona. The first space charge measurement was performed 2 min after the sample completed corona charging as the sample had to be transferred to the PEA setup. The bulk charge is not evident when a short charging time is adopted. However, the bulk charge is clearly seen when the charging time is extended to $10 \mathrm{~min}$ as shown in figure $4(b)$. It can also be seen that a small amount of negative charge is present across the sample. This may serve as evidence that charge injection from the top has taken place.

Figure 4 also shows that the charge decay rate is different for different charging times. Charge decay is more rapid when a longer charging time is adopted. As the amount of charge in the sample is closely related to the surface potential measured, this observation is consistent with the results shown in figure 3.

When the corona charging voltage increases, charge injection becomes more obvious as shown in figure 5 where a $-8 \mathrm{kV}$ grid voltage was applied. Compared with figure 4 where a lower corona charging voltage was used, the second and third peaks (corresponding to the bottom and top surfaces of the sample) in the PEA measurement become broader towards the bulk of the sample, indicating the presence of bulk charge. These bulk charges can only be injected into the sample from the corresponding surface. It is clear that both positive and negative charges are present in the bulk of the sample. When the charging time is extended to 10 min significant positive charge injection can be observed, as shown in figure $5(b)$. It is worth noting that the scale for charge density reflects rapid decay of charge within the first $2 \mathrm{~min}$. The charge decay rate increases significantly compared with the sample charged at $2 \mathrm{kV}$. This result is in agreement with the cross-over phenomenon observed in figure 2 . The sample arrangement for the PEA measurement differs from that in the surface potential measurement. The two added layers and induced charge on the electrodes may affect the charge movement and therefore, the charge decay rate. Nevertheless, our early work on comparison between the surface potential obtained through either the PEA measurement or direct surface potential measurement indicating little difference, suggested the added two layers have insignificant influence on the charge transport.

To further verify the occurrence of bipolar charge injection in the corona-charged sample, two layers of $50 \mu \mathrm{m}$ films were corona charged for $2 \mathrm{~min}$ with a grid voltage of $-8 \mathrm{kV}$. The interface of LDPE is known to be able to trap both positive and negative charges due to surface states. The injected positive and negative charges can be captured by the interface during 


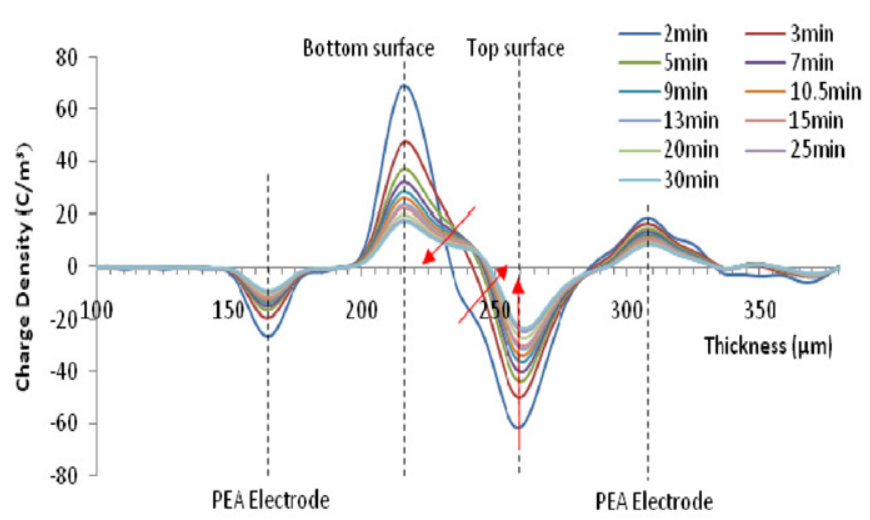

(a) $t_{c}=2$ minutes

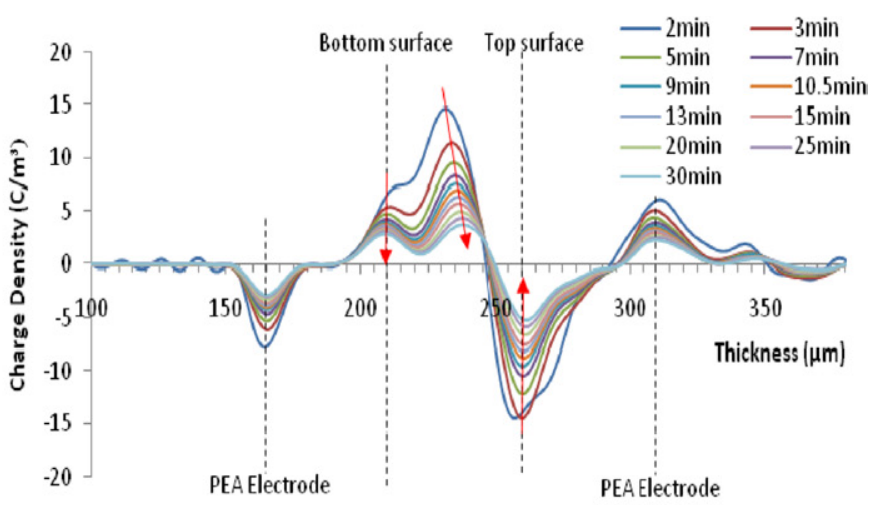

(b) $t_{c}=10$ minutes

Figure 5. Space charge distribution in a $50 \mu \mathrm{m}$ sample corona charged at $-8 \mathrm{kV}$ for $(a) 2 \mathrm{~min}$ and $(b) 10 \mathrm{~min}$.

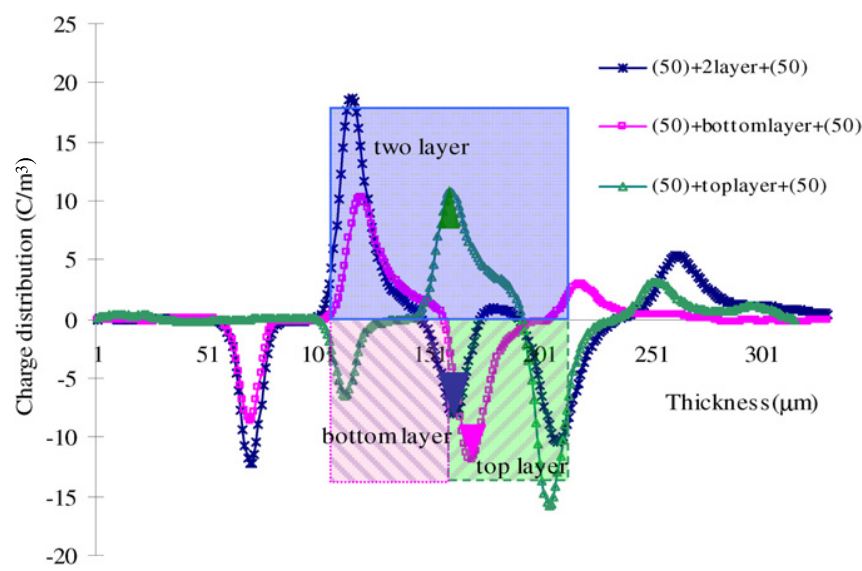

Figure 6. Space charge distribution for different layers of corona-charged sample ( $-8 \mathrm{kV}$ and $2 \mathrm{~min})$.

the transport process. To observe these trapped charges, space charge measurements were performed on the two-layered corona-charged sample first and then on both the top and the bottom layers separately. The results are shown in figure 6 , where two $50 \mu \mathrm{m}$ LDPE films were attached to both sides of each layer. From these distributions it is evident that negative charge is present at the top surface of the bottom layer (bottom layer curve or pink curve) and positive charge at the bottom surface of the top layer (top layer curve or green curve). The amount of negative charge is greater than that of positive charge, so overall it shows a negative charge peak (2layer curve or blue curve). The revelation of both positive and negative charges at the interface between the top and the bottom layer is a clear indication of bipolar charge injection. In this instance, positive charge tends to move upwards and therefore is trapped at the bottom surface of the top layer. Similarly, negative charge is trapped at the top surface of the bottom layer.

\section{New model and preliminary simulation result}

Recently, the bipolar charge injection model has been used extensively to simulate charge transport, trapping and recombination under de voltage. The simulation results show

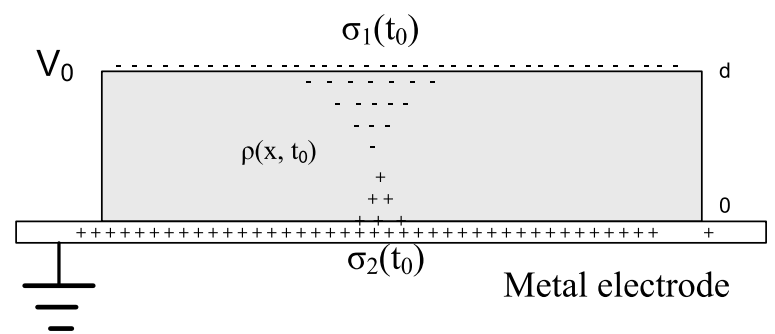

Figure 7. Schematic diagram of surface charge and space charge distribution immediately after corona charging.

a good match with experimental results. Considering the new evidence of bipolar charge injection during the corona charging, we reckon the model can be applied to explain the surface potential decay with some modifications. The new modified model takes bipolar injection into consideration. Additionally, a tunnelling process has been proposed to account for charge injection from the top surface while the conventional Schottky injection is used for the bottom surface.

Based on the space charge measurement from the PEA, the initial charge distribution immediately after the corona charging in a sample can be illustrated in figure 7 .

Here $V_{0}$ is the grid voltage. $\sigma_{1}\left(t_{0}\right), \rho\left(x, t_{0}\right)$ and $\sigma_{2}\left(t_{0}\right)$ are surface charge on the top surface, space charge in the sample and induced charge on the metal electrode, respectively. The initial values will depend on the grid voltage and charging time. They all change with time leading to a change in surface potential $V(t)$.

The principle of all space charge models lies in the description of the charge conduction and electrical transport mechanism across the material. The Alison-Hill [23] model aims to effectively describe the bipolar transport and space charge phenomena in solid dielectrics under high de stress. The bipolar transport is described as a conduction process governed by an effective mobility. This feature distinguishes the model from the others. In effect, charge carriers are injected from the electrodes, electrons from the cathode and holes from the anode. Injection occurs based on the Schottky mechanism [24] 


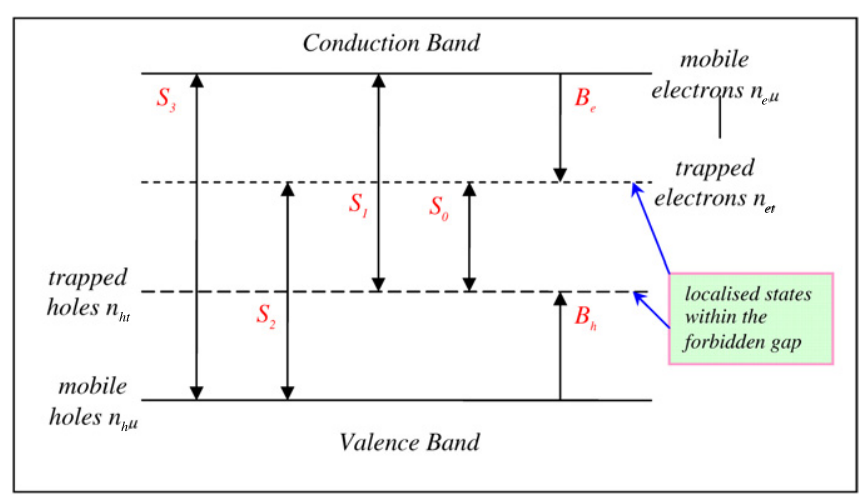

Figure 8. Schematic representation of the conduction and trapping model. $S_{\mathrm{i}}$ are recombination coefficients, and $n_{e \mu}, n_{e \mathrm{t}}, n_{h \mathrm{t}}, n_{h \mu}$ are mobile and trapped electron and hole densities. $B_{\mathrm{e}}$ and $B_{\mathrm{h}}$ are electron and hole trapping coefficients.

whereby overcoming a potential barrier $W$ at the interfaces:

$$
J_{s}=A T^{2} \exp \left(-\frac{W-\sqrt{e^{3} E / 4 \pi \varepsilon_{\mathrm{r}} \varepsilon_{0}}}{k T}\right),
$$

where $A$ is a constant related to the material, $T$ the temperature, $k$ the Boltzmann constant, $e$ the electron charge, $E$ the electric field at the interface, $\varepsilon_{\mathrm{r}}$ the relative permittivity of the material and $\varepsilon_{0}$ the permittivity of vacuum.

It can be seen that the potential barrier is lowered because of the application of the electric field. This will lead to an increase in the injected current.

After penetrating into the material, the carriers, under the influence of the applied field, will drift across the material characterized by an effective mobility. Throughout its motion, some carriers are trapped in the localized states, i.e. deep trap centres and therefore the total amount of charges moving across reduces. However, no extraction barrier is introduced in the model but, on the other hand, they are prone to recombine with their opposite species (electrons with holes).

Due to the fact that oppositely charged species, electrons and holes are being considered in the numerical computation, charge trapping and mutual annihilation or recombination between these species were introduced into the model. The model now contains four species of charge particles, namely, mobile electrons $\left(e_{\mu}\right)$ and holes $\left(h_{\mu}\right)$, and immobile electrons $\left(e_{\mathrm{t}}\right)$ and holes $\left(h_{\mathrm{t}}\right)$ (at trap sites) as shown in figure 8.

Charge transportation in solid dielectrics is essentially governed by a set of basic equations. They describe the behaviour of charge carriers in the system through a time and space dependent total flux $j(x, t)$ and by neglecting diffusion [23]:

Transport equation:

$$
j_{\mathrm{C}}(x, t)=\mu n(x, t) E(x, t),
$$

Continuity equation:

$$
\frac{\partial n(x, t)}{\partial t}+\frac{\partial j(x, t)}{\partial x}=s,
$$

Poisson's equation:

$$
\frac{\partial E(x, t)}{\partial x}=\frac{\rho(x, t)}{\varepsilon},
$$

where $\mu$ is the mobility of carriers, $n$ the density of mobile species, $E$ the electric field, $j$ the current density, $x$ the spatial coordinate, $t$ the time, $s$ the source term, $\varepsilon$ the dielectric permittivity and $\rho$ the net charge density.

The above model has been widely used for simulation of space charge build up and dynamics, and achieved a satisfactory match when the parameters were appropriately selected [25]. It has also been used to model transient current and anomalous discharge current successfully [26].

For a corona-charged sample, once charges are injected into the bulk, the rest description of charge trapping, detrapping, recombination and transport can be readily applied using the existing bipolar model.

It has been well accepted that the mobility of charge carriers is a function of the electric field. In fact, several models have attempted to use field-dependent mobility to account for the cross-over phenomenon without success. The fielddependent mobility can also be easily implemented in the proposed model. A power law proposed by Wintle [16] has been given as

$$
\mu=c E^{n-1} \quad n \geqslant 1,
$$

where $c$ is a constant and $n$ is a fixed component. Both of them are material dependent.

The charge transport in the bulk of the sample is determined by the electric field. The electric field in the sample at any time consists of contributions from the three components, i.e. space charge $\rho(x, t)$, surface charge density at the top $\sigma_{1}(t)$ and the induced surface charge density at the bottom electrode $\sigma_{2}(t)$. Let us assume the field components are represented by $E_{\rho}(t), E_{\sigma_{1}}(t)$ and $E_{\sigma_{2}}(t)$, respectively. The surface potential across the sample can be calculated by integrating the total electric field:

$$
V_{0}(t)=\int_{0}^{d}\left[E_{\rho}(t)+E_{\sigma_{1}}(t)+E_{\sigma_{2}}(t)\right] \mathrm{d} x .
$$

In addition, the total charge in the system at any time must be in balance, i.e.

$$
\sigma_{1}(t) S+\sigma_{2}(t) S+\int_{0}^{d} \rho(x, t) S \mathrm{~d} x=0,
$$

where $S$ is the surface area where charges are present.

Clearly, $\sigma_{1}(t), \sigma_{2}(t)$ and $\rho(x, t)$ are not independent quantities. Based on the modified model, it is possible to calculate $\rho(x, t)$ during the corona charging until a predefined charging time $t=t_{0}$. The quantities $V_{0}\left(t_{0}\right)$ and $\rho\left(x, t_{0}\right)$ are the initial condition for surface potential decay. This allows one to determine $\sigma_{1}\left(t_{0}\right)$ and $\sigma_{2}\left(t_{0}\right)$ using the above two equations.

Once these initial four quantities are determined, one can calculate new space charge distribution $\rho\left(x, t_{0}+\Delta t\right)$ based on the proposed model and the two surface density $\sigma_{1}\left(t_{0}+\Delta t\right)$ and $\sigma_{2}\left(t_{0}+\Delta t\right)$ using the Fowler-Nordheim (FN) tunnelling 


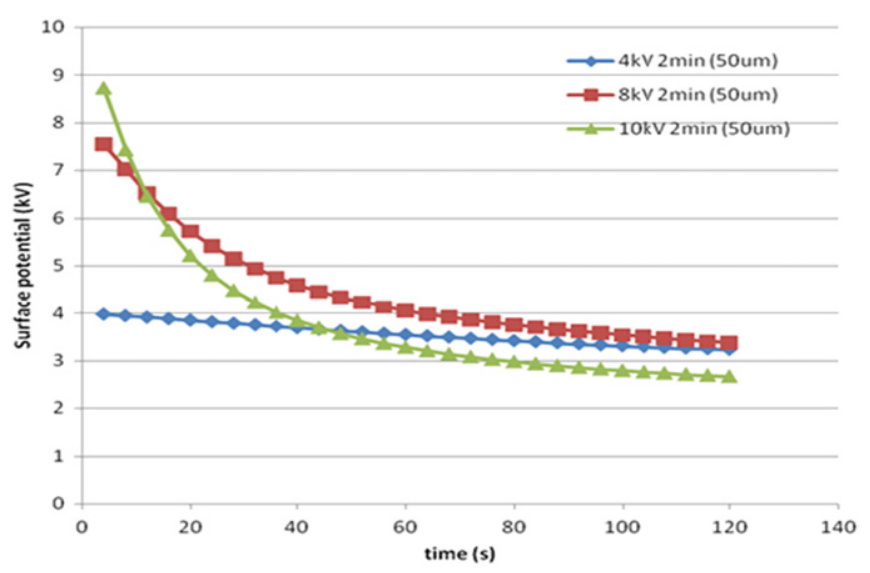

Figure 9. Initial simulation showing cross-over phenomenon in surface potential decay (power-law mobility: $\mu=4.5 \times 10^{-16} E^{n-1}$, $n=1.15)$

and Schottky injection, respectively. In addition, as the system is an open circuit, the injected charge must satisfy the following condition:

Maxwell's equation for the total current:

$$
J(t)=j_{\mathrm{C}}(x, t)+\varepsilon \frac{\partial E(x, t)}{\partial t}=0 .
$$

The surface potential at time $t=t_{0}+\Delta t$ can finally be computed using equation (6). Repeating the above process, the relationship between the surface potential and time can be calculated.

Our initial simulation work shows it is possible to achieve surface potential cross-over provided the initial surface potential and space charge distribution are set right as shown in figure 9. In this simulation the parameters are similar to those used in [25]. The only difference here is that the mobility equation (5) has been utilized in this study instead of a constant mobility. Additionally, the phenomenon presented in figure 3 is readily explained by the modified model as a long charging time will result in more charge injection and less surface charge densities for both top and bottom. The charge distribution favours the charge recombination and therefore a rapid surface potential decay. In the present simulation, Schottky injections have been used for both surfaces. Schottky injection typically describes the injection from metal to semiconductor or insulator, so the FN tunnelling may be more appropriate for the corona-charged surface. More simulation is under way where the FN tunnelling will be investigated and it is important to point out that the present model allows one to extract much more material information.

\section{Conclusions}

Based on the new experimental evidence from the space charge measurements on the corona-charged LDPE film, a modified bipolar charge injection model has been proposed to account for surface charge decay. The new model can take care of fielddependent carrier mobility and readily explains the surface potential cross-over phenomenon and surface potential decay with different charging times.
More simulations based on the proposed model will be carried out and the results will be compared with the experiments.

\section{References}

[1] Ieda M, Sawa G and Shinohara I 1967 A decay process of surface electric charges across polyethylene film J. Appl. Phys. 6 793-4

[2] Jestin P, Coelho R and Goldman M 1987 Surface potential decay on epoxy resin Inst. Phys. Conf. Ser. 85 193-6

[3] Baum E A, Lewis T J and Toomer R 1978 The lateral motion of charge on thin films of polyethylene terephthalate J. Phys. D: Appl. Phys. 11 963-77

[4] Mady F, Renoud R and Ganachaud J P 2002 Modelling of potential decay experiment. Influence of the microscopic charge transport processes on the macroscopic observable phenomena 3rd Conf. French Society of Electrostatics (Toulouse, France 2002)

[5] Berlepsch H V 1985 Interpretation of surface potential kinetics in HDPE by a trapping model J. Phys. D: Appl. Phys. $181155-70$

[6] Molinié P 1995 Surface potential decay on corona-charged epoxy samples due to polarization processes J. Phys. D: Appl. Phys. 28 1601-10

[7] Chen G, Xu Z and Zhang L W 2007 Measurement of the surface potential decay of corona-charged polymer films using the pulsed electroacoustic method Meas. Sci. Technol. 18 1453-8

[8] Xu Z, Zhang L and Chen G 2007 Decay of electric charge on corona charged polyethylene J. Phys. D: Appl. Phys. 40 7085-9

[9] Salhi S, Bellal A, Ziari Z, Kahlouche A and Segui Y 2003 Measure and analysis of potential decay in polypropylene films after negative corona charge deposition J. Electrostat. 57 169-81

[10] Sonnonstine T J and Perlman M M 1975 Surface potential decay in insulators with field dependent mobility and injection efficiency J. Appl. Phys. 41 3975-81

[11] Batra I P, Keiji Kanazawa K and Seki H 1970 Discharge characteristics on photoconducting insulators J. Appl. Phys. $413416-22$

[12] Batra I P, Keiji Kanazawa K, Schechtman B H and Seki H 1971 Charge carrier dynamics following pulsed photoinjection J. Appl. Phys. 42 1124-30

[13] Seki H and Batra I P 1971 Photocurrents due to pulse illumination in the presence of trapping: II J. Appl. Phys. 42 2407-20

[14] Wintle H J 1970 Decay of static electrification by conduction processes in polyethylene J. Appl. Phys. 41 4004-7

[15] Wintle H J 1971 Decay of surface electric charge in insulators Japan. J. Appl. Phys. 10 659-60

[16] Wintle H J 1972 Surface-charge decay in insulators with non-constant mobility and with deep trapping J. Appl. Phys. $432927-30$

[17] Batra I P and Keiji Kanazawa K 1972 On the 'cross-over' effect in surface potential decay Japan. J. Appl. Phys. 11 267-8

[18] Baum E A, Lewis T J and Toomer R 1977 Decay of electrical charge on polyethylene films J. Phys. D: Appl. Phys. 10 487-97

[19] Baum E A, Lewis T J and Toomer R 1977 Further observations on the decay of surface potential of corona charged polyethylene films J. Phys. D: Appl. Phys. 10 2525-31

[20] Mizutani T, Oota A and Ieda M 1978 Surface potential decay of corona-charged high-density polyethylene Japan. J. Appl. Phys. 17 2157-8 
[21] Kao K J, Bamji S S and Perlman M M 1979 Thermally stimulated discharge current study of surface charge release in polyethylene by corona generated excited molecules, and the crossover phenomenon J. Appl. Phys. 50 8181-5

[22] Toomer R and Lewis T J 1980 Charge trapping in corona-charged polyethylene films J. Phys. D: Appl. Phys. $131343-56$

[23] Alison J M and Hill R M 1994 A model for bipolar charge transport trapping and recombination in degassed crosslinked polyethylene J. Phys. D: Appl. Phys. 27 1291-9
[24] Kao K C and Hwang W 1981 Electrical Transport in Solids (Oxford: Pergamnon)

[25] Chen G and Loi S H 2005 Space charge modelling in solid dielectrics under high electric field based on double charge injection model MRS Fall Meeting (Boston, MA)

[26] Roy S L, Segur P, Teyssedre G and Laurent C 2004 Description of bipolar charge transport in polyethylene using a fluid model with a constant mobility: model prediction J. Phys. D: Appl. Phys. 37 298-305 